\title{
Fluid flow induced by nonuniform ac electric fields in electrolytes on microelectrodes. II. A linear double-layer analysis
}

\author{
A. González \\ Departamento de Física Aplicada, ESI University of Seville, Camino de los Descubrimientos, s/n 41092 Sevilla, Spain \\ A. Ramos, * N. G. Green, and A. Castellanos \\ Departamento de Electrónica y Electromagnetismo, University of Seville, Avenida Reina Mercedes, s/n 41012 Sevilla, Spain \\ H. Morgan \\ Department of Electronics and Electrical Engineering, University of Glasgow, Glasgow G12 8LT, Scotland, United Kingdom
}

(Received 12 August 1999)

\begin{abstract}
Frequency-dependent fluid flow in electrolytes on microelectrodes subjected to ac voltages has recently been reported. The fluid flow is predominant at frequencies of the order of the relaxation frequency of the electrodeelectrolyte system. The mechanism responsible for this motion has been termed ac electro-osmosis: a continuous flow driven by the interaction of the oscillating electric field and the charge at the diffuse double layer on the electrodes. This paper develops the basis of a theoretical approach to this problem using a linear double layer analysis. The theoretical results are compared with the experiments, and a good correlation is found.
\end{abstract}

PACS number(s): 82.70.Dd, 47.65.+a, 82.45.+z, 85.90.+h

\section{INTRODUCTION}

In recent publications the experimental observation of a new type of fluid flow in electrolytes on microelectrodes energized with ac electric fields has been reported, a phenomenon termed ac electro-osmosis [1-3]. The presence of a highly divergent ac electric field on an electrode induces a fluid flow across the electrode surface. A detailed series of experiments were performed in order to characterize the fluid behavior as a function of frequency, voltage, and conductivity, and the results were presented in our previous paper [4]. The direction of the flow is always from the electrode edge, onto the surface of the electrode. The magnitude of the fluid velocity is frequency dependent, tending to zero at low and high frequency limits. The fluid velocity peaks at a characteristic frequency which is a function of medium conductivity and distance from the electrode edge [4].

The fluid flow is assumed to have its origin in the interaction of the electric field and the induced free charge in the double layer on the electrodes. A simple circuit model [3] was developed to account for the experimental results, and was found to qualitatively explain the observed flow [4]. However, in order to quantitatively describe the fluid velocity, an analysis from first principles is required. Recent publications also reported movement of particles close to electrode surfaces, and attributed the movement to fluid flow (due to electrical forces in the double layer) rather than electrical forces acting directly on the particles [5,6].

In this paper a linear analysis of ac electro-osmosis in a geometry similar to the experimental electrodes is presented. First, some assumptions and simplifications are made using physical aspects of the experiments. This leads to the decoupling of the electrical problem from the fluid problem. The

\footnotetext{
*Author to whom correspondence should be addressed. Electronic address: ramos@cica.es
}

electrical problem is then studied using the thin double-layer approximation. The electrohydrodynamic fluid equations are examined, and the ac electro-osmotic velocity derived. The theoretical results are then compared with the experimental data, demonstrating a good correlation. Finally, some conclusions are drawn, and further refinements to the model are discussed.

\section{Basic assumptions and simplifications}

The experimental system consisted of two coplanar rectangular electrodes $2 \mathrm{~mm}$ long and $100 \mu \mathrm{m}$ wide, separated by a $25 \mu \mathrm{m}$ gap [4]. Aqueous solutions of $\mathrm{KCl}$ were used because of the symmetry, both in valency and mobility, of the $\mathrm{K}^{+}$and $\mathrm{Cl}^{-}$ions.

The electrode length is much greater than the width. The system can therefore be simplified to two dimensions, as shown in Fig. 1. The $x$ axis lies parallel to the electrode surface and perpendicular to the gap, with $x=0$ defined to be at the center of the gap. The $y$ axis is perpendicular to the electrode surface, with $y=0$ defined to be at the surface. In order to obtain an analytical solution, the electrodes are assumed to be semi-infinite with an infinitely small gap. This assumption produces a singularity at $x=0$ and $y=0$, and

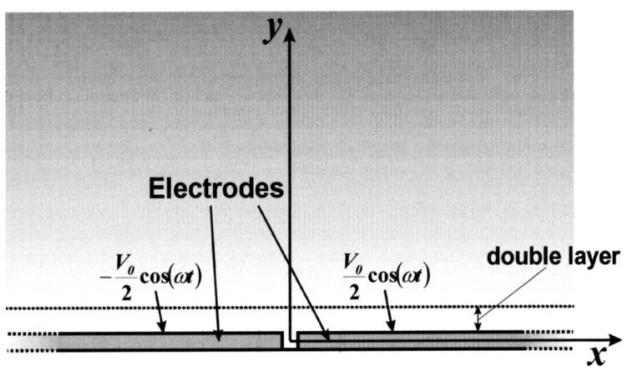

FIG. 1. Diagram illustrating the geometry used in the theoretical model.

4019

(C) 2000 The American Physical Society 
incorrect values at large $x$. However, only the solution at intermediate distances, which correspond to the physical dimensions of the electrodes, is considered.

The equations which describe the system are further simplified by considering a symmetrical electrolyte. This is a reasonable consideration since the difference in the mobilities of the $\mathrm{K}^{+}$and $\mathrm{Cl}^{-}$ions is approximately $4 \%$ [7].

The electrodes are assumed to be ideally polarizable, i.e., the free charge flow from the electrodes to the electrolyte is neglected. Chemical reactions at the electrode surface can be an important consideration in the determination of the electrode polarization. However, measurements of the electrode impedance show that the impedance grows as the frequency decreases, implying that ideally polarizable electrodes may be a valid simplification. Therefore, this paper concentrates on developing a theory based solely on the electrolyte response.

The fixed surface potential of the electrodes and the applied ac potential are assumed to be small, so that a linear approximation can be made. As a result, an analytical solution can be obtained to explain the experimental observations, and to suggest approaches for a generalization of the equations to the nonlinear case. Although in the experiments [4] the potential drop across the diffuse double layer is probably much greater than $k_{B} T / e \simeq 25 \mathrm{mV}$ [8], nevertheless insights into the phenomena can be obtained from the linear analytical solution.

Finally, the convection current is assumed to be much smaller than the conduction current induced by the applied electric field. For typical experimental conditions [4] and for a field of $10^{5} \mathrm{~V} / \mathrm{m}$, the ion velocity is of order $1 \mathrm{~cm} / \mathrm{s}$ and the observed liquid velocities are lower than $0.05 \mathrm{~cm} / \mathrm{s}$. In fact, the ratio between convection and conduction currents is even smaller, since the conduction current is greater in the double layer, where the charge density is not negligible. As a result, the convection current is neglected in the calculation of the electrical current density. Therefore, the electrical problem can be solved independently of the velocity field and the solution inserted into the equations for the fluid motion.

\section{ELECTRICAL PROBLEM}

\section{A. Basic equations}

The electric potential $\phi$ is related to the positive and negative ion densities $n_{+}$and $n_{-}$by Poisson's equation:

$$
\nabla^{2} \phi=\frac{e\left(n_{-}-n_{+}\right)}{\epsilon},
$$

and two ion number conservation equations

$$
\frac{\partial n_{ \pm}}{\partial t}+\boldsymbol{\nabla} \cdot \mathbf{J}_{ \pm}=0
$$

where the ion density currents $\mathbf{J}_{ \pm}$are given by

$$
\mathbf{J}_{ \pm}=\mp n_{ \pm} \mu \boldsymbol{\nabla} \phi-D \nabla n_{ \pm}+n_{ \pm} \mathbf{u} \text {. }
$$

$u$ is the liquid velocity, $e$ is the electronic charge, $\epsilon$ is the permittivity of the fluid, and $\mu$ and $D$ are the mobility and diffusion coefficients of the ions, respectively. The ionic flux [Eq. (3)] consists of three terms: electric drift, diffusion, and drift due to the fluid motion. The last term is the convection current, which is neglected as discussed previously.

These equations have the following boundary conditions. At the electrodes $(y=0)$, the electric potential is a combination of the static surface potential, $\psi_{0}$, and an imposed alternating voltage of amplitude $V_{0}$ :

$$
\phi= \begin{cases}\psi_{0}+\frac{1}{2} V_{0} \cos \omega t & \text { if } x>0 \\ \psi_{0}-\frac{1}{2} V_{0} \cos \omega t & \text { if } x<0\end{cases}
$$

We have assumed that the imposed voltage does not affect the surface potential.

Since the electrodes are ideally polarizable, the normal components of the ion density currents vanish at the electrodes

$$
\mathbf{n} \cdot \mathbf{J}_{ \pm}=\mp n_{ \pm} \mu \frac{\partial \phi}{\partial y}-D \frac{\partial n_{ \pm}}{\partial y}=0 .
$$

Finally, as $y \rightarrow \infty$, the electric field vanishes and the ion densities tend to their equilibrium values:

$$
n \pm \rightarrow n_{0}, \quad \phi \rightarrow 0 \quad(y \rightarrow \infty)
$$

\section{B. Scales and parameters}

For convenience the equations will be made nondimensional. First, two linear combinations of the ion densities, the charge density $(\rho)$ and the mean ion density $(n)$, are defined:

$$
\rho=e\left(n_{+}-n_{-}\right) \quad n=\frac{n_{+}+n_{-}}{2} .
$$

The magnitude of each variable can then be scaled as follows:

$$
y=\left(\frac{\epsilon D}{\sigma}\right)^{1 / 2} y^{\prime}=\lambda_{D} y^{\prime}, \quad x=L x^{\prime}, \quad t=\frac{t^{\prime}}{\omega},
$$

$$
\phi=\frac{D}{\mu} \phi^{\prime}=\frac{k_{B} T}{e} \phi^{\prime}, \quad \rho=2 n_{0} e \rho^{\prime}, \quad n=n_{0} n^{\prime},
$$

where $\sigma=2 n_{0} e \mu$ is the conductivity, $n_{0}$ is the ion number density of the bulk fluid, $\omega$ is the applied frequency, $k_{B}$ is Boltzmann's constant, and $T$ is the absolute temperature. Since the rates of change of each variable are very different, the spatial coordinates are scaled using two distinct quantities. For the $y$ axis the (static) Debye length $\lambda_{D}$, the typical scale for the decay of charge density, is used. For the $x$ axis, a length $L$ is used, a scale characteristic of the experiments that reflects the changes in the potential along the electrodes. For the experimental setup described in our previous paper, $L$ is approximately 1000 times greater than $\lambda_{D}$.

Using these scales, the system depends on four dimensionless parameters:

$$
\omega^{\prime}=\frac{\omega \epsilon}{\sigma}, \quad \delta=\frac{\lambda_{D}}{L}, \quad V_{0}^{\prime}=\frac{V_{0} e}{k_{B} T}, \quad \phi_{0}^{\prime}=\frac{\psi_{0} e}{k_{B} T} .
$$


The parameter $\delta$ is small, of order $\frac{1}{1000}$ in this case. This allows the thin layer approximation $\delta \ll 1$ to be used $[9,10]$.

The resulting nondimensional equations are (dropping the primes):

$$
\begin{gathered}
\frac{\partial^{2} \phi}{\partial y^{2}}+\delta^{2} \frac{\partial^{2} \phi}{\partial x^{2}}=-\rho, \\
\omega \frac{\partial \rho}{\partial t}-\frac{\partial}{\partial y}\left(\frac{\partial \rho}{\partial y}+n \frac{\partial \phi}{\partial y}\right)-\delta^{2} \frac{\partial}{\partial x}\left(\frac{\partial \rho}{\partial x}+n \frac{\partial \phi}{\partial x}\right)=0, \\
\omega \frac{\partial n}{\partial t}-\frac{\partial}{\partial y}\left(\frac{\partial n}{\partial y}+\rho \frac{\partial \phi}{\partial y}\right)-\delta^{2} \frac{\partial}{\partial x}\left(\frac{\partial n}{\partial x}+\rho \frac{\partial \phi}{\partial x}\right)=0,
\end{gathered}
$$

with boundary conditions

$$
\begin{gathered}
\phi \rightarrow 0, \quad \rho \rightarrow 0, \quad n \rightarrow 1 \quad(y \rightarrow \infty), \\
\frac{\partial \rho}{\partial y}+n \frac{\partial \phi}{\partial y}=0, \quad \frac{\partial n}{\partial y}+\rho \frac{\partial \phi}{\partial y}=0 \quad(y=0), \\
\phi= \begin{cases}\psi_{0}+\frac{1}{2} V_{0} \cos t & \text { if } x>0 \\
\psi_{0}-\frac{1}{2} V_{0} \cos t & \text { if } x<0 .\end{cases}
\end{gathered}
$$

\section{Linear approximation}

The complete system is nonlinear, and its solution is not trivial. To linearize it, both $\psi_{0}$ and $V_{0}$ are assumed to be small quantities. In this limit, both the electric potential and the charge density are similarly small. The mean ion density differs only slightly from unity (its value far from the electrodes), and can be written as $n=1+c$, with $c \ll 1$. In the linear system the superposition principle can be applied, and each function written as the sum of a static and an oscillating part. The static part is denoted by a subscript $s$.

The static equations reduce to those for a simple double layer in the Debye-Hückel approximation

$$
\begin{gathered}
\frac{\partial^{2} \phi_{s}}{\partial y^{2}}+\delta^{2} \frac{\partial^{2} \phi_{s}}{\partial x^{2}}=-\rho_{s}, \\
\left(\frac{\partial^{2}}{\partial y^{2}}+\delta^{2} \frac{\partial^{2}}{\partial x^{2}}\right)\left(\rho_{s}+\phi_{s}\right)=0, \\
\frac{\partial^{2} c_{s}}{\partial y^{2}}+\delta^{2} \frac{\partial^{2} c_{s}}{\partial x^{2}}=0,
\end{gathered}
$$

with boundary conditions

$$
\begin{gathered}
\phi_{s} \rightarrow 0, \quad \rho \rightarrow 0, \quad c_{s} \rightarrow 0 \quad(y \rightarrow \infty), \\
\phi_{s}=\psi_{0}, \quad \frac{\partial}{\partial y}\left(\rho_{s}+\phi_{s}\right)=0, \quad \frac{\partial c_{s}}{\partial y}=0 \quad(y=0) .
\end{gathered}
$$

The solution of this system is simply

$$
\phi_{s}=\psi_{0} e^{-y}, \quad \rho=-\psi_{0} e^{-y}, \quad c_{s}=0 .
$$

This result shows that the static potential and charge density depend only on the $y$ coordinate. This distribution does not produce stresses in the liquid, and can therefore be ignored.

For the oscillating part of the system, complex amplitudes can be used instead of time-dependent functions, i.e., $f$, where a general function is $f(t)=\operatorname{Re}(f \exp (i \omega t))$. From the boundary conditions it can be concluded that both the electric potential and the charge density are odd functions of $x$, and we restrict ourselves to the region $x>0$.

The equations are

$$
\begin{gathered}
\frac{\partial^{2} \phi}{\partial y^{2}}+\delta^{2} \frac{\partial^{2} \phi}{\partial x^{2}}=-\rho, \\
i \omega \rho-\frac{\partial^{2}}{\partial y^{2}}(\rho+\phi)-\delta^{2} \frac{\partial^{2}}{\partial x^{2}}(\rho+\phi)=0,
\end{gathered}
$$

with boundary conditions

$$
\begin{gathered}
\rho \rightarrow 0, \quad \phi \rightarrow 0 \quad(y \rightarrow \infty), \\
\frac{\partial}{\partial y}(\rho+\phi)=0, \quad \phi=\frac{V_{0}}{2} \quad(y=0),
\end{gathered}
$$

and the decoupled density equation

$$
i \omega c-\frac{\partial^{2} c}{\partial y^{2}}-\delta^{2} \frac{\partial^{2} c}{\partial x^{2}}=0,
$$

with boundary conditions

$$
c \rightarrow 0 \quad(y \rightarrow \infty), \quad \frac{\partial c}{\partial y}=0 \quad(y=0) .
$$

The solution for the second problem is simply

$$
c=0 .
$$

This result and the decoupling of the equations imply that the typical diffusion length scale $\omega^{-1 / 2}$ disappears from this problem. This is a consequence of the assumption of a symmetrical electrolyte and linearity. In general $[10,11]$, the equations cannot be decoupled.

\section{Thin double-layer approximation}

The linear equations for $\rho$ and $\phi$ [Eqs. (23) and (24)] can be completely solved for the prescribed boundary conditions (see the Appendix). However, it is more useful to use an approximate version derived using the matched asymptotic expansion method (also used, in a similar context, in Ref. [12]) since this is suitable for generalization to other electrode geometries.

In this approximation there are two different length scales. The small scale, corresponding to the Debye length, over which the charge density goes from its maximum value, close to the electrodes, to zero, corresponding to the bulk. The electrical stress is concentrated in distances of this order above the electrodes. The large scale is "macroscopic," corresponding to the characteristic dimension of the electrodes and the variations of the electric field. This scale describes the tangential field that acts on the charge density. The ratio of these two scales is the previously defined quantity $\delta$. 
Two scaled coordinates can then be defined: the "inner" $y$ (to be used near the electrodes, where $y \sim 1$ ), and the "'outer" $Y=\delta y$ (to describe the bulk, where $y \sim 1 / \delta$ ). The corresponding inner and outer electric potential functions will be denoted by $\phi$ and $\Phi$.

The inner and outer charge densities are denoted by $\rho$ and $R$. As will be shown, the solution for the inner density $\rho$ is an exponentially decreasing function. The outer charge density therefore obeys a linear equation with homogeneous boundary conditions at infinity and at the outer limit of the double layer (since it must match an exponentially small function). As a result, the solution is $R=0$ and only the inner charge density needs to be considered.

The equations are second order, and terms up to first order in $\delta$ must be retained to match inner and outer solutions. The equations for the inner problem are

$$
\begin{gathered}
\frac{\partial^{2} \phi}{\partial y^{2}}=-\rho, \\
\frac{\partial^{2} \rho}{\partial y^{2}}=(1+i \omega) \rho,
\end{gathered}
$$

with the boundary conditions at $y=0$

$$
\phi=\frac{V_{0}}{2}, \quad \frac{\partial}{\partial y}(\rho+\phi)=0 .
$$

For the outer problem, the only equation is Laplace's equation

$$
\frac{\partial^{2} \Phi}{\partial Y^{2}}+\frac{\partial^{2} \Phi}{\partial x^{2}}=0
$$

The matching conditions require the solutions to be tangent up to first order in $\delta$ in the overlapping region. Therefore,

$$
\begin{gathered}
\lim _{y \rightarrow \infty} \frac{\partial \phi}{\partial y}=\delta \lim _{Y \rightarrow 0} \frac{\partial \Phi}{\partial Y}, \\
\lim _{y \rightarrow \infty}\left(\phi-y \frac{\partial \phi}{\partial y}\right)=\lim _{Y \rightarrow 0}\left(\Phi-Y \frac{\partial \Phi}{\partial Y}\right), \\
\lim _{y \rightarrow \infty} \rho=0, \quad \lim _{y \rightarrow \infty} \frac{\partial \rho}{\partial y}=0 .
\end{gathered}
$$

The solution for the inner problem is

$$
\rho=A e^{-s y}, \quad \phi=-\frac{A e^{-s y}}{s^{2}}+C y+D,
$$

where

$$
s=\sqrt{1+i \omega}, \quad \operatorname{Re}(s)>0 .
$$

Imposing the boundary conditions at $y=0$ gives

$$
D=\frac{V_{0}}{2}+\frac{A}{1+i \omega}, \quad C=\frac{i \omega A}{\sqrt{1+i \omega}} .
$$

For the outer problem the solution must now satisfy the matching conditions

$$
\begin{aligned}
& \lim _{Y=0} \Phi=D=\frac{V_{0}}{2}+\frac{A}{1+i \omega}, \\
& \lim _{Y=0} \frac{\partial \Phi}{\partial Y}=\frac{C}{\delta}=\frac{i \omega}{\delta \sqrt{1+i \omega}} A .
\end{aligned}
$$

A boundary condition for the outer potential at $Y=0$ can be obtained by eliminating $A$ from the previous two equations:

$$
\Phi-\frac{1}{i \Omega} \frac{\partial \Phi}{\partial Y}=\frac{V_{0}}{2},
$$

where

$$
\Omega=\frac{\omega \sqrt{1+i \omega}}{\delta} .
$$

The second term in Eq. (42) is the potential drop across the double layer. Therefore, the boundary condition for the outer potential is simply the sum of the applied voltage and the potential drop. For low frequencies, below the charge relaxation frequency, this potential drop is equal to that for a capacitance per unit area of value $\epsilon / \lambda_{D}$. As a result, a circuit model can be used to represent the whole system [3]. However, this circuit is not enough to calculate the forces, since details about the structure of the double layer are required.

For the outer potential, Laplace's equation can be solved using the Fourier sine transform

$$
\Phi(x, Y)=\int_{0}^{\infty} \widetilde{\Phi}(k, Y) \sin k x d k
$$

which results in

$$
\Phi(x, Y)=\frac{i \Omega V_{0}}{\pi} \int_{0}^{\infty} \frac{e^{-k Y} \sin k x}{k(k+i \Omega)} d k .
$$

Near the electrodes, this reduces to

$$
\Phi(x, 0)=\frac{i \Omega V_{0}}{\pi} \int_{0}^{\infty} \frac{\sin k x}{k(k+i \Omega)} d k=\frac{V_{0}}{2}(1-F(\Omega x)),
$$

with

$$
F(p)=\frac{2}{\pi} \int_{0}^{\infty} \frac{\sin u}{u+i p} d u=e^{-p}+\frac{i}{\pi}\left(e^{p} \operatorname{Ei}(-p)-e^{-p} \operatorname{Ei}(p)\right),
$$

and $\operatorname{Ei}(p)$ is the exponential integral

$$
\operatorname{Ei}(p)=-\mathcal{P} \int_{-p}^{\infty} \frac{e^{-t}}{t} d t
$$

(where $\mathcal{P}$ denotes Cauchy's principal value.)

Once the outer potential is known, complete expressions for the inner problem can be written. The complex amplitude for the charge density is 


$$
\rho=-\frac{V_{0}}{2}(1+i \omega) F(\Omega x) e^{-s y},
$$

and the inner electric potential is

$\phi=\frac{V_{0}}{2} e^{-s y}+\frac{V_{0}}{2}(1-F(\Omega x))\left(1-e^{-s y}\right)-i \delta \Omega \frac{V_{0}}{2} F(\Omega x) y$.

\section{E. Inclusion of the Stern layer}

In general, models for the double layer include a compact layer of relatively immobile ions at the electrode surface. In the simplest Gouy-Stern model for the double layer, the compact layer consists of hydrated ions and the potential varies linearly, giving a fixed capacitance per unit area. In addition, the inclusion of an extra capacitance means that it is possible to analyze a system of electrodes covered with a layer of insulator $\left(\mathrm{Si}_{3} \mathrm{~N}_{4}\right)$ [2].

Consider a compact layer with mean height $h$ in units of $\lambda_{D}$ and mean permittivity $\epsilon_{c}$ in units of $\epsilon$. Since $h$ is very small, the oscillating part of the potential in the compact layer can be written as a linear function of the coordinate $y$ :

$$
\phi_{c}=\frac{V_{0}}{2}+B(y+h) .
$$

Here $B$ is a constant given by the boundary conditions, and the electrode is now located at $y=-h$, so that the diffuse double layer still starts at $y=0$. The ions in the Stern layer are assumed to be immobile, so that there is no free charge flow to the diffuse layer due to the oscillating potential. The displacement current is therefore continuous at $y=0$, and the new boundary condition for the electric potential $\phi$ in the diffuse layer at $y=0$ can be written as

$$
\phi-\frac{\partial \phi}{\partial y} \frac{h}{\epsilon_{c}}=\frac{V_{0}}{2} .
$$

Normally what is obtained experimentally is the capacitance per unit area for the compact layer $\epsilon_{c} / h$ [8].

Taking Eq. (52) into account, the asymptotic matching conditions give a boundary condition for the outer potential at $Y=0$, which can still be written as Eq. (42) where $\Omega$ is now given by

$$
\Omega=\frac{\omega \sqrt{1+i \omega}}{\delta} \frac{1}{1+\frac{h}{\epsilon_{c}}(1+i \omega)^{3 / 2}} .
$$

The charge density and potential in the diffuse double layer are then

$$
\rho=-\frac{V_{0}}{2} F(\Omega x) \frac{s^{2} e^{-s y}}{1+\frac{h}{\epsilon_{c}} s^{3 / 2}},
$$

$$
\phi=\frac{V_{0}}{2} F(\Omega x) \frac{e^{-s y}}{1+\frac{h}{\epsilon_{c}} s^{3 / 2}}+\frac{V_{0}}{2}(1-F(\Omega x))
$$

$$
-i \omega \frac{V_{0}}{2} F(\Omega x) \frac{s y}{1+\frac{h}{\epsilon_{c}} s^{3 / 2}} .
$$

\section{F. Generalization to other electrode geometries}

This method can be generalized to the case of more complicated electrodes and three-dimensional problems, provided that the double layer width is much smaller than the typical dimension of the electrodes. In this case, Laplace's equation should be solved in the large-scale domain using the mixed boundary condition given in Eq. (42). The asymptotic matching conditions, together with the boundary conditions for the inner problem at $y=0$, can be used to give a solution for the inner potential and charge density as functions of the potential drop across the double layer:

$$
\begin{gathered}
\phi=-\frac{\delta \Omega}{\omega s}\left(\Phi-\frac{V_{0}}{2}\right) e^{-s y}+i \delta \Omega\left(\Phi-\frac{V_{0}}{2}\right) y+\Phi, \\
\rho=\frac{s \delta \Omega}{\omega}\left(\Phi-\frac{V_{0}}{2}\right) e^{-s y} .
\end{gathered}
$$

Here $\Omega$ is given by Eqs. (43) or (53) depending on whether or not the Stern layer is included. Given the charge density and the potential in the diffuse layer, the electrical stress on the fluid can be calculated.

\section{LIQUID MOTION}

The ac electro-osmotic flow arises from the stress produced by the interaction of the electric field and the charge density, balanced by viscous friction. As the charge density is nonzero only inside the double layer, the electrical stress is determined by the inner solution. In the linear limit, both the charge density and the electric field are harmonic oscillating functions. The product of these functions gives the electric stress, which has a nonzero time average. Therefore, there is a continuous flow in the ac regime.

There is an alternating motion superimposed on this continuous flow, resulting from the coupling between the static and oscillating solutions. The frequency of this motion is the same as the applied voltage (in the range $10^{2}-10^{5} \mathrm{~Hz}$ ) and practically unobservable in the experiments [4].

The liquid motion is governed by the incompressibility and Navier-Stokes equations

$$
\boldsymbol{\nabla} \cdot \mathbf{u}=0
$$

$$
\rho_{m} \frac{D \mathbf{u}}{D t}=-\nabla p+\rho \mathbf{E}+\eta \nabla^{2} \mathbf{u}
$$

where $u$ is the liquid velocity, $\mathbf{E}$ the electric field, $\rho_{m}$ the mass density, $\eta$ the dynamic viscosity and $p$ the pressure. The steady flow is given by the time average of these equations. 
For microelectrodes, a typical length is $L \sim 2 \times 10^{-5} \mathrm{~m}$, the maximum velocity is $u \sim 5 \times 10^{-4} \mathrm{~m} / \mathrm{s}$, and the kinematic viscosity is $\nu=\eta / \rho_{m}=10^{-6} \mathrm{~m}^{2} / \mathrm{s}$ [4]. Therefore, the Reynolds number can be estimated to be at maximum of the order of $u L / \nu \sim 10^{-2}$, and the fluid can be considered to be noninertial. In this limit, the time-averaged equations are

$$
\begin{gathered}
\frac{\partial u}{\partial x}+\frac{\partial w}{\partial y}=0 \\
0=-\frac{\partial p}{\partial x}-\left\langle\rho \frac{\partial \phi}{\partial x}\right\rangle+\eta\left(\frac{\partial^{2} u}{\partial x^{2}}+\frac{\partial^{2} u}{\partial y^{2}}\right), \\
0=-\frac{\partial p}{\partial y}-\left\langle\rho \frac{\partial \phi}{\partial y}\right\rangle+\eta\left(\frac{\partial^{2} w}{\partial x^{2}}+\frac{\partial^{2} w}{\partial y^{2}}\right),
\end{gathered}
$$

where $u$ and $w$ denote the components of the liquid velocity tangential and normal to the electrode surface, respectively.

The liquid motion is governed by the electrical stress and the pressure gradient. Using the same scales as before [Eqs. (8) and (9)], the scales for the pressure and liquid velocity can be deduced from Eqs. (60) and (61) as

$$
p_{0}=2 n_{0} k_{B} T, \quad u_{0}=\frac{2 n_{0} k_{B} T \lambda_{D}^{2}}{\eta L}, \quad w_{0}=\frac{\lambda_{D}}{L} u_{0},
$$

resulting in the nondimensional equations

$$
\begin{gathered}
\frac{\partial u}{\partial x}+\frac{\partial w}{\partial y}=0 \\
-\frac{\partial p}{\partial x}-\left\langle\rho \frac{\partial \phi}{\partial x}\right\rangle+\frac{\partial^{2} u}{\partial y^{2}}+\delta^{2} \frac{\partial^{2} u}{\partial x^{2}}=0, \\
-\frac{\partial p}{\partial y}-\left\langle\rho \frac{\partial \phi}{\partial y}\right\rangle+\delta^{2} \frac{\partial^{2} w}{\partial y^{2}}+\delta^{4} \frac{\partial^{2} w}{\partial x^{2}}=0 .
\end{gathered}
$$

For $\delta \ll 1$, the normal velocity terms can be neglected in Eq. (66). This is a consequence of the incompressibility equation (60) and the null normal velocity condition at the electrodes.

The time average of the product of two harmonic functions, described by complex amplitudes, is

$$
\langle f g\rangle=\frac{1}{T} \int_{0}^{T} f(t) g(t) d t=\frac{1}{2} \operatorname{Re}\left(f g^{*}\right),
$$

where $g *$ indicates the complex conjugate of $g$. Therefore, from Eq. (66) the pressure distribution is

$$
\frac{\partial p}{\partial y}=-\left\langle\rho \frac{\partial \phi}{\partial y}\right\rangle=-\frac{1}{2} \operatorname{Re}\left(\rho \frac{\partial \phi^{*}}{\partial y}\right) .
$$

Applying Poisson's equation in the diffuse layer, and integrating, gives

$$
p=p_{0}+\frac{1}{4}\left|\frac{\partial \phi}{\partial y}\right|^{2}
$$

Substituting this pressure distribution into the tangential equation (65) gives

$$
\frac{\partial^{2} u}{\partial y^{2}}=\frac{\partial p}{\partial x}+\frac{1}{2} \operatorname{Re}\left(\rho \frac{\partial \phi^{*}}{\partial x}\right) .
$$

The expressions for stress and liquid velocity can be simplified if $\omega \ll 1$. The experiments show that the maximum velocity is observed when $\omega$ is of the order of $\delta$ (in dimensional form, $\left.\omega \sim \sigma \lambda_{D} / \epsilon x\right)$ [4]. In addition, this restriction can be justified by considering that $\omega \sim 1$ or greater implies $\Omega \gg 1$, from Eqs. (43) and (53). From the boundary condition (42), $\Omega \gg 1$ implies that

$$
\left(\Phi-\frac{V_{0}}{2}\right) \rightarrow 0 \quad \text { at } Y=0,
$$

i.e., there is no potential dropped across the double layer and the charge density tends to zero, as does the electrical stress. As a result, the problem can be restricted to the regime where $\omega \ll 1$.

In this case,

$$
\Omega \simeq \frac{\omega}{\delta} \Lambda
$$

where $\Lambda=1$ for the simple diffuse layer and $\Lambda=1 /(1$ $\left.+h / \epsilon_{c}\right)$ including the Stern layer. Neglecting terms of first order in $\delta$, the potential and charge density in the diffuse layer are given by

$$
\begin{gathered}
\phi \simeq \frac{V_{0}}{2} F(\Omega x) \Lambda e^{-y}+\frac{V_{0}}{2}(1-F(\Omega x)), \\
\rho \simeq-\frac{V_{0}}{2}(\Omega x) \Lambda e^{-y} .
\end{gathered}
$$

The pressure distribution reduces to

$$
p=p_{0}+\frac{V_{0}^{2}}{16} \Lambda^{2}|F(\Omega x)|^{2} e^{-2 y},
$$

and the equation for the tangential velocity (70) becomes

$$
\frac{\partial^{2} u}{\partial y^{2}}=\frac{\partial p}{\partial x}+\frac{1}{2} \operatorname{Re}\left(\rho \frac{\partial \phi^{*}}{\partial x}\right)=\frac{V_{0}^{2}}{16} \Lambda e^{-y} \frac{\partial}{\partial x}\left(|F(\Omega x)|^{2}\right) .
$$

Integrating this equation twice and applying the boundary conditions of null velocity at $y=0$ and null derivative at $y$ $\rightarrow \infty$ gives the ac electro-osmotic velocity outside the double layer,

$$
U=-\frac{V_{0}^{2}}{16} \Lambda \frac{\partial}{\partial x}|F(\Omega x)|^{2}
$$

or, in dimensional form,

$$
U=-\frac{\epsilon V_{0}^{2}}{16 \eta} \Lambda \frac{\partial}{\partial x}\left|F\left(\Lambda \frac{\epsilon \omega x}{\sigma \lambda_{D}}\right)\right|^{2},
$$

where now $\Lambda=1$ for the simple diffuse layer and $1 /(1$ $\left.+h \epsilon / \lambda_{D} \epsilon_{c}\right)$ when including the Stern layer. According to this expression, the effect of the Stern layer is to reduce the 


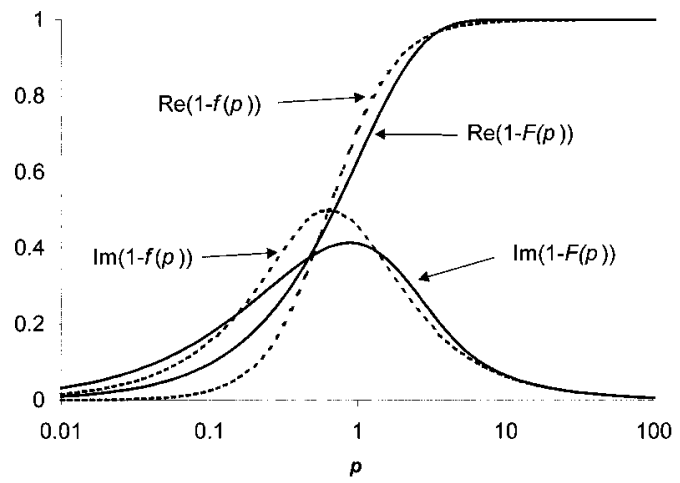

FIG. 2. Calculated normalized potential drop across the bulk electrolyte as a function of the nondimensional frequency. The solid lines represent the real and imaginary parts of $1-F(p)$, and the dashed lines represent the corresponding functions for $1-f(p)$.

velocity by the factor $\Lambda$ and increase the frequency of maximum velocity by the same factor.

As discussed in Sec. IIF, a general form of Eq. (78) can be obtained for other electrode geometries, provided that the double layer width is much smaller than the typical dimension of the electrodes and the linear approximation still applies. From the potential and charge density expressions, the electro-osmotic velocity can be derived as

$$
\mathbf{U}=-\frac{\epsilon}{4 \eta} \Lambda \nabla_{s}\left(|\Delta V|^{2}\right),
$$

where $\Delta V$ is the potential drop across the double layer and $\nabla_{s}$ denotes a gradient across the electrode surface. According to this expression, the liquid tends to move from regions with a high potential drop, and therefore a high charge density, to regions where the potential drop is lower. Typically, this is from strong to weak electric field regions on the electrodes. This has been observed in experiments.

\section{COMPARISON OF THEORY AND EXPERIMENTS}

\section{A. Potential drop across the double layer}

Measuring the impedance of the system as a function of frequency provides information about the polarization of the double layer. In the experiments [4], the impedance was analyzed to give the average potential drop across the double layer, normalized to the applied potential.

From the linear model the normalized potential drop across the double layer for a given position and frequency is

$$
\frac{\Delta V}{V_{0}}=\frac{F(p)}{2},
$$

with $F(p)$ defined in Eq. (47) and $p=\Lambda \epsilon \omega x / \sigma \lambda_{D}$. Taking a semicircular path from one electrode to the other, $F(p)$ represents the normalized potential dropped across both double layers and $1-F(p)$ represents the normalized potential dropped across the bulk. This last function is shown in Fig. 2 (solid line). The real part of the normalized potential drop reaches a value of 0.5 at $p=0.693$, while the imaginary part has a maximum of 0.414 at $p=0.879$. Clearly, the figure shows that, for a given position, the potential is entirely

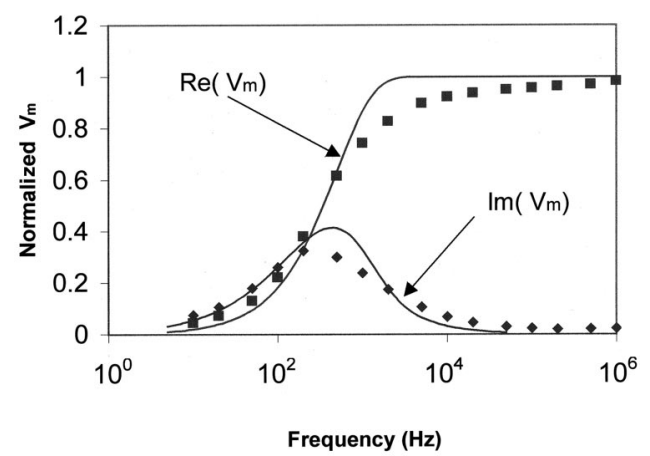

FIG. 3. Comparison of the normalized potential drop across the bulk, $V_{m}$, as predicted from the diffuse layer model (solid line) together with the experimental data (points).

dropped across the double layers at low frequencies, and that it is dropped across the bulk at high frequencies.

This figure also shows an equivalent function derived from the circuit model given in Ref. [3] and used in our previous paper for comparison (dotted line). Here the system is modeled with the bulk electrolyte represented as a series of resistors following semicircular paths, terminated at either end by a distributed capacitor, representing the double layer. The surface charge density and the tangential electric field are estimated from the potential drop in the capacitors. This results in an analog of the function $\Phi$ at $Y=0$ [Eq. (46)] which is

$$
V(x)=\frac{V_{0}}{2}(1-f(p)), \quad p=\frac{\Lambda \epsilon \omega x}{\sigma \lambda_{D}},
$$

where

$$
f(p)=\frac{1}{1+i p \pi / 2}
$$

in the units used in this paper. Comparing the functions, $f(p)$ and $F(p)$ (Fig. 2), it can be seen that they are similar, implying that the circuit model gives a good picture of the electrical problem. In the circuit model, both the point at which the real part is 0.5 and the maximum in the imaginary part occur at $p=2 / \pi$, with a value of 0.5 for the maximum. The function $F(p)$ shows a greater dispersion than that derived from the circuit model, $f(p)$.

In dimensional form and for the simple diffuse layer case $(\Lambda=1)$, the frequency scales as

$$
\omega=\frac{\sigma \lambda_{D}}{\epsilon x} p
$$

Since $\lambda_{D}$ is proportional to $\sigma^{-1 / 2}$ [Eq. (8)], the position of the maximum of the imaginary parts are proportional to the square root of the conductivity. Experimentally, although the frequency of maximum increases with conductivity, the $\sigma^{1 / 2}$ dependence is not observed [4].

Measurements of the impedance presented in our previous paper were analyzed to give the normalized average of the potential drop across the bulk electrolyte. Figure 3 shows the normalized potential drop across the bulk calculated from the linear theory (solid line) together with the experimental data, 


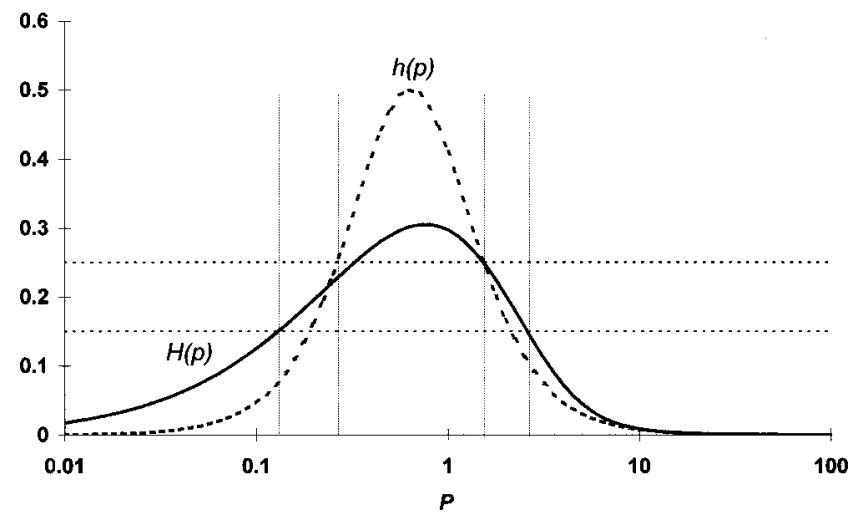

FIG. 4. Plot of the nondimensional velocity as a function of nondimensional frequency. The solid line corresponds to the linear diffuse layer model, and the dashed line to the circuit model. The horizontal and vertical lines mark the points of half-height.

for an electrolyte of conductivity $\sigma=0.0086 \mathrm{~S} / \mathrm{m}$. For the calculation $x$ was chosen to be $50 \mu \mathrm{m}$, corresponding to a point in the middle of the electrodes. While the correlation is good, the experiments show a broader relaxation process than is predicted by the linear theory.

\section{B. Liquid motion}

Equation (78) gives an estimate for the ac electro-osmotic velocity just outside the double layer, at distances of the order of the Debye length. The velocity measurements were taken at a much greater distance [4], approximately $1 \mu \mathrm{m}$ above the electrode surface. The velocity at this distance is described by a solution of the complete fluid motion equation, which in the absence of external forces reduces to the biharmonic equation for the stream function, $\left(\nabla^{2}\right)^{2} \psi=0$. The boundary conditions are the ac electro-osmotic velocity $U$ at $Y=0$, and nondivergence far from the electrodes. For solutions of the biharmonic equation (as for Laplace's equation) the horizontal and vertical scales are of the same order. Since the horizontal scale is around $25 \mu \mathrm{m}$, the velocity at 1 $\mu \mathrm{m}$ is expected to be comparable to $U$, except close to the edge of the electrodes. Therefore, it is valid to compare the experimental data with Eq. (78).

The ac electro-osmotic velocity can be written as

$$
U=\frac{\epsilon V_{0}^{2}}{16 \eta x} H(p)
$$

for the case $\Lambda=1$, with

$$
H(p) \equiv p \frac{\partial}{\partial p}\left(|F(p)|^{2}\right), \quad p=\frac{\epsilon \omega x}{\sigma \lambda_{D}}
$$

For a given value of $x$, the velocity $U$ is proportional to the nondimensional function $H(p)$, which is shown in Fig. 4. One of the important characteristics of the fluid motion observed in our previous paper was that distance and frequency appeared to be linked by one parameter. As the linear theory clearly shows for this particular geometry, this is indeed the case. The theoretical velocity plot has a bell shape, similar to the experimental results, where, for a given position, the frequency of the maximum velocity occurs at $p=0.761$, with
TABLE I. Experimental and predicted frequencies (in $\mathrm{Hz}$ ) for the maximum velocity at a distance of $x=22.5 \mu \mathrm{m}$ from the center of the gap: (a) diffuse layer only; (b) Stern layer included.

\begin{tabular}{cccc}
\hline \hline$\sigma(\mathrm{S} / \mathrm{m})$ & $f_{\max }($ Expt. $)$ & $f_{\max }(a)$ & $f_{\max }(b)$ \\
\hline $2.1 \times 10^{-3}$ & 175 & 405 & 460 \\
$8.6 \times 10^{-3}$ & 730 & 820 & 1048 \\
$8.4 \times 10^{-2}$ & 4770 & 2563 & 4793 \\
\hline \hline
\end{tabular}

$H=0.305$. The bell width, defined as the mean width at half height, can also be determined. The predicted width in decades for the parameter $p$ and also for the frequency is

$$
\Delta(\log p)=\Delta(\log f)=1.28 \text {. }
$$

The experimental value lies in the range 1.2-1.5.

The circuit model can also be used to estimate the velocity of liquid motion, using a formula analogous to Eq. (83):

$$
U=\frac{\epsilon V_{0}^{2}}{16 \eta x} h(p),
$$

where

$$
h(p) \equiv p \frac{\partial}{\partial p}\left(|f(p)|^{2}\right), \quad p=\frac{\epsilon \omega x}{\sigma \lambda_{D}},
$$

and $f(p)$ is given by Eq. (81). Equation (85) predicts a velocity which varies with conductivity and voltage in the same manner as for the diffuse layer model. However, in order to obtain Eq. (85), a volume charge distribution in the diffuse layer must be assumed. In previous work [3], the diffuse layer charge was modeled as a charged surface situated at the Debye length. This model coincidentally leads to a similar result as that of the diffuse layer model. From inspection of function $h(p)$, the maximum velocity occurs at $p=0.637$, which corresponds to a lower frequency than predicted by the diffuse layer model. The maximum velocity is predicted to be one and a half times greater than for the diffuse layer model, and with a bell width of 0.766 decades, narrower than for the diffuse layer model and the experimental data.

For an aqueous solution $\left(\epsilon \simeq 7 \times 10^{-10} \mathrm{~F} / \mathrm{m}\right.$ and $\eta$ $\simeq 10^{-3} \mathrm{~kg} / \mathrm{m} \mathrm{s}$ ), the frequency (in $\mathrm{Hz}$ ) of maximum velocity is

$$
f_{\max }=\Lambda^{-1} \frac{0.199 \sqrt{\sigma}}{x},
$$

where the experimental mobilities for the ions $\mathrm{K}^{+}$and $\mathrm{Cl}^{-}$ [7] have been used. For $\Lambda=1$, Eq. (87) predicts a dependence with the square root of the conductivity, which is not observed experimentally. The frequency of maximum fluid velocity for the three different electrolytes (see our previous paper) is summarized in Table I. These data were measured for a point at distance of $x=22.5 \mu \mathrm{m}$ from the center of the gap. Also shown in the table are the frequencies of maximum velocity calculated from Eq. (87) without a Stern layer (a) and with a Stern layer (b). For the latter case the capacitance of the Stern layer was set to $20 \mu \mathrm{F} \mathrm{cm}^{-2}$, a typical value for a compact layer $[8,14]$. The table indicates that $f_{\max }$ increases with medium conductivity, and for the highest con- 
ductivity the inclusion of the Stern layer clearly has an influence on the frequency. Theoretically, $f_{\max }$ is directly proportional to the relaxation frequency of the double layerelectrolyte impedance: this is observed experimentally [4].

For an aqueous solution, the predicted maximum velocity at position $x$ is

$$
U_{\max }=\frac{1.368 \times 10^{-8} V_{0}^{2}}{x}
$$

for the case $\Lambda=1$. This equation predicts a peak velocity that is independent of conductivity and proportional to the voltage squared. For an applied voltage of $0.5 \mathrm{~V}$ and at a distance of $22.5 \mu \mathrm{m}$ from the center of the system, it predicts a velocity of $1.5 \times 10^{-4} \mathrm{~m} / \mathrm{s}$. For conductivities of 2.1 and $8.6 \times 10^{-3} \mathrm{~S} / \mathrm{m}$, the corresponding measured velocity was $0.35 \times 10^{-4} \mathrm{~m} / \mathrm{s}$, four times smaller than the predicted value. For 1 and $2.5 \mathrm{~V}$ it was observed that the magnitude of the peak velocity varied with conductivity. This could be due to nonlinear effects in the diffuse double layer. For $0.5 \mathrm{~V}$, a weak dependence on conductivity was observed (see Fig. 7 of the previous paper).

For the lowest conductivity electrolyte the velocity was approximately proportional to the voltage squared. This was not observed for the other two conductivities.

\section{CONCLUSIONS AND FURTHER DEVELOPMENTS}

A linear double layer analysis of ac electro-osmosis for perfectly polarizable electrodes has been carried out and applied to a geometry similar to the experimental system outlined in our previous paper. A thin double-layer approximation has been used to simplify the equations and an expression for the ac electro-osmotic velocity has been derived.

Theoretical predictions have been compared with the experimental results presented in our previous paper. A well defined pattern of fluid flow, moving from high to low electric field regions (from the electrode edge to the center) was observed in the experiments, as predicted. The theoretical frequency and distance dependence of velocity was similar to the experimental results. The order of magnitude of the velocity was in reasonable agreement for low voltages and conductivities. Both the experimental data and the theoretical model indicate a correlation between the frequency dependence of the velocity and the voltage drop across the double layer.

However, the model does not account for the variation of the velocity magnitude with conductivity as observed experimentally, neither does it account for the frequency dependence of peak velocity on conductivity. There are several possible explanations for this. A nonlinear analysis of the diffuse double layer may account for the conductivity dependence of the velocity magnitude. For low frequencies, the structure of the diffuse layer may be similar to the GouyChapman solution for a static double layer, with time dependent parameters. In this case, as the potential across the double layer increases the charge is pulled closer to the electrode. This reduces the effective Debye length and may reduce the fluid velocity.

In addition, in this work it was assumed that the elec- trodes were ideally polarizable. In reality, faradaic current flows between the electrodes and the electrolyte, a system usually modeled as a resistance in parallel with the double layer impedance $[13,14]$. A fuller description of the double layer impedance is likely to give more accurate values for the frequencies of peak velocity. Alternatively, since the ac electro-osmotic velocity is related to the voltage drop across the double layer, the liquid velocity may be deduced from experimental measurements of the double-layer impedance.

\section{ACKNOWLEDGMENTS}

This work was supported by the Spanish DGES under Contract No. PB96-1375. N. G. G. also received financial support from The European Union through Contract No. BIO4-CT98-5010.

\section{APPENDIX: COMPLETE SOLUTION OF THE LINEAR ELECTRICAL PROBLEM}

It is possible to solve the electrical problem in the linear limit completely without making the thin double-layer approximation. Although not easily extensible to other electrode configurations, this solution can be used to test the approximate solution presented in the text.

The equations to be solved are Eqs. (23) and (24), subjected to boundary conditions (25) and (26). Due to the antisymmetry of the boundary conditions, we can deduce that both the charge and the potential are odd functions of $x$. In this case, we can introduce the Fourier sine transforms

$$
\begin{aligned}
& \widetilde{\phi}(k, y)=\frac{2}{\pi} \int_{0}^{\infty} \phi(x, y) \sin (k x) d x, \\
& \widetilde{\rho}(k, y)=\frac{2}{\pi} \int_{0}^{\infty} \rho(x, y) \sin (k x) d x .
\end{aligned}
$$

This transform reduces the system to a set of ordinary differential equations

$$
\begin{gathered}
\frac{d^{2} \widetilde{\rho}}{d y^{2}}=\left(1+i \omega+\delta^{2} k^{2}\right) \widetilde{\rho}, \\
\frac{d^{2} \widetilde{\phi}}{d y^{2}}=k^{2} \delta^{2} \widetilde{\phi}-\widetilde{\rho},
\end{gathered}
$$

with the boundary conditions

$$
\begin{gathered}
\tilde{\phi} \rightarrow 0, \quad \widetilde{\rho} \rightarrow 0 \quad(y \rightarrow \infty), \\
\frac{d}{d y}(\widetilde{\rho}+\widetilde{\phi})=0, \quad \widetilde{\phi}=\frac{V_{0}}{\pi k} \quad(y=0) .
\end{gathered}
$$

The inverse Fourier transform of the solution to this system is

$$
\begin{gathered}
\rho=-V_{0} \int_{0}^{\infty} \frac{\delta(s+\delta k)}{\pi\left(s^{2}-1+\delta k s\right)} e^{-s y} \sin (k x) d k, \\
\phi=V_{0} \int_{0}^{\infty} \frac{\left(i \omega e^{-\delta k y}+\delta k e^{-s y}\right)}{\pi k(\delta k-s)\left(s^{2}-1+\delta k s\right)} \sin k x d k,
\end{gathered}
$$


with

$$
s=\sqrt{1+i \omega+\delta^{2} k^{2}}, \quad \operatorname{Re}(s)>0 .
$$

This solution is valid for all values of $\omega$.

The expression for the voltage just outside the double layer is obtained by letting $y \rightarrow \infty$, while $\delta y=0$. In this case, from Eq. (A8) the relative voltage drop across both double layers [analogous to $F(p)]$ is

$$
\frac{V_{0}-2 \Phi}{V_{0}}=1-\int_{0}^{\infty} \frac{i \omega}{\pi k(\delta k-s)\left(s^{2}-1+\delta k s\right)} \sin k x d k
$$

Contrary to the case of the thin layer limit, the complete expression depends not only on the product $\omega x$, but on $x$ and $\omega$, independently. In Fig. 5 the exact results for $\delta=0.001$ with $x=0.001$ and 1 (the more realistic case), are compared with the thin layer approximation. Recalling that $\delta$ is the ratio between typical scales, a value of $x=1$ means that the point is placed at a distance 1000 times the Debye length from the center, while $x=0.001$ means that it is placed at one Debye length from the center. The figure shows that for val-

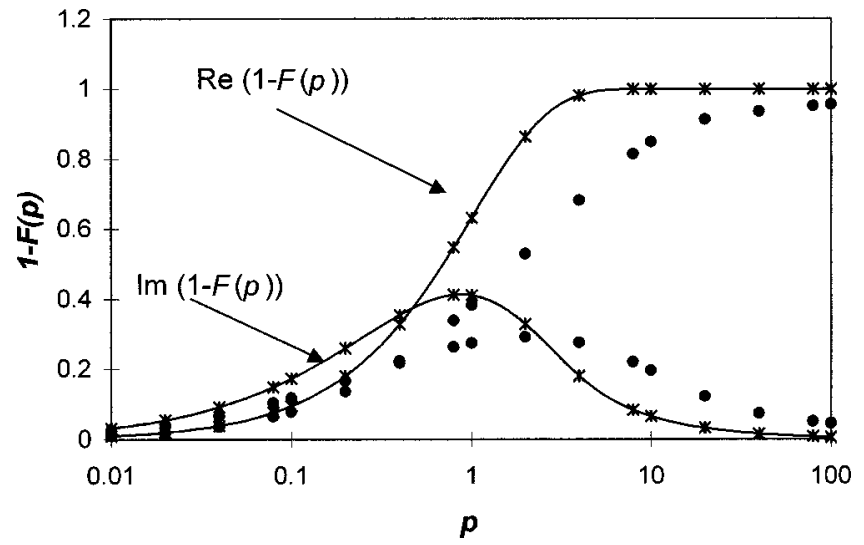

FIG. 5. Comparison of the voltage drop across the bulk in the thin layer limit (solid line), with the exact solution for half-infinite plates, for $x=1$ (stars) and $x=0.001$ (circles).

ues of $x$ of order unity the complete solution and the thin layer approximation solution are fully in accordance. The figure also shows that the relevant parameter is $\omega x / \delta$ and, for a given position with finite $x$, the characteristic frequency is of the order of $\delta$.
[1] A. Ramos, H. Morgan, N. G. Green, and A. Castellanos, J. Phys. D 31, 2388 (1998).

[2] A. Ramos, H. Morgan, N. G. Green, and A. Castellanos, J. Electrost. 47, 71 (1999).

[3] A. Ramos, H. Morgan, N. G. Green, and A. Castellanos, J. Colloid Interface Sci. 217, 420 (1999).

[4] N. G. Green, A. Ramos, A. González, H. Morgan, and A. Castellanos, previous paper, Phys. Rev. E. 61, 4011 (2000).

[5] M. Trau, D. A. Saville, and I. A. Aksay, Langmuir 13, 6375 (1997).

[6] S.-R. Yeh, M. Seul, and B. I. Shraiman, Nature (London) 386, 57 (1997).

[7] CRC Handbook of Chemistry and Physics, 74th ed., edited by D. R. Lide (CRC Press, London, 1994).
[8] R. J. Hunter, Zeta Potential in Colloid Science (Academic, San Diego, 1981).

[9] R. W. O’Brien, J. Colloid Interface Sci. 92, 204 (1995).

[10] W. C. Chew and P. N. Sen, J. Chem. Phys. 77, 4683 (1982).

[11] E. H. B. DeLacey and L. R. White, J. Chem. Soc. Faraday Trans. 2 78, 457 (1982).

[12] J. Gunning, D. Y. C. Chan, and L. R. White, J. Colloid Interface Sci. 170, 522 (1995).

[13] M. Sluyters-Rehbach and J. H. Sluyters, in Electroanalytical Chemistry, edited by A. J. Bard (Dekker, New York, 1970), Vol. 4, Chap. 1.

[14] J. O. M. Bockris and A. K. N. Reddy, Modern Electrochemistry (Plenum, New York, 1973). 